\title{
Cathodal tDCS increases stop-signal reaction time
}

\author{
Maximilian A. Friehs ${ }^{1}$ (D) $\cdot$ Christian Frings $^{1}$
}

Published online: 16 July 2019

(C) The Psychonomic Society, Inc. 2019

\begin{abstract}
Transcranial direct current stimulation (tDCS) is a noninvasive method of modulating human brain activity and potentially alters performance in cognitive tasks. Often it is assumed that effects of tDCS modulation depend on the polarity - anodal stimulation typically boost cognitive processes whereas cathodal stimulation hampers them. While most tDCS research focusses on the effects of anodal stimulation, cathodal tDCS effects are underexplored. In the present study, cathodal tDCS over the right dorsolateral prefrontal cortex (rDLPFC) was used to potentially hamper the response inhibition process as measured by the stop-signal task (SST). A $9 \mathrm{~cm}^{2}$ cathode was always positioned over the rDLPFC while the $35 \mathrm{~cm}^{2}$ anode was placed over the left deltoid. We contrasted a cathodal stimulation condition (that is assumed to reduce neural processing) with sham stimulation and expected a decrease in SST performance after cathodal tDCS, as evidenced by an increase in stop-signal reaction time (SSRT). In a sample of $N=45$ healthy adults, a significant Time $\times$ tDCS condition interaction emerged, indicating an increase in SSRT after cathodal tDCS. In a recent study by Friehs and Frings (Journal of Experimental Psychology: Human Perception and Performance, 2018), using a similar study design and stimulation protocol, single-session anodal tDCS over the rDLPFC was used to enhance SST performance as indicated by an acceleration of SSRT. In concert, these results suggest that response inhibition is tied to the neural state of the rDLPFC.
\end{abstract}

Keywords Cognitive control $\cdot$ Prefrontal cortex $\cdot$ tDCS $\cdot$ Neural network $\cdot$ Stop-Signal Task

Stopping an already initialized action is an ability essential for adaptive everyday behavior. For example, a person might have to stop moving in order to avoid a collision with a car, or a baseball player might have to stop on the base because the baseman caught the ball midair. Withholding a response in order to achieve a goal (e.g., not getting injured or being safe in the baseball game) is crucial for our life. The stop-signal task (Logan, Cowan, \& Davis, 1984) is used to study this kind of response inhibition. The task requires participants to react to a stimulus, but inhibit the already-initiated action if a stop signal is presented after the stimulus onset.

Results from studies on patients - for example, with PFC lesions (Aron, Fletcher, Bullmore, Sahakian, \& Robbins, 2003) or ADHD (Lijffijt, Kenemans, Verbaten, \& Van Engeland, 2005) - show that there are considerable differences between individuals in their ability to inhibit a

Maximilian A. Friehs

friehs@uni-trier.de

1 Cognitive Psychology, University of Trier, Trier, Germany response after it has been initiated. But such differences can occur not only between individuals but also within an individual, meaning that an individual's abilities are not set in stone from birth onwards. Exemplary, an increased efficiency of the inhibition process can be observed after intense training (Kramer et al., 1999; Tsai, 2009; Verbruggen, Logan, \& Stevens, 2008; Wang et al., 2013). Furthermore, methods such as noninvasive brain stimulation techniques (e.g., transcranial direct current stimulation, or tDCS for short) can potentially be used to modulate cognitive processes within an individual. While anodal tDCS typically is associated with facilitatory effects, cathodal tDCS - although typically linked to inhibitory effects - has shown inconsistent results (Schroeder \& Plewnia, 2017). Accordingly, findings from neuromodulation studies using anodal tDCS suggest that the response inhibition process can be boosted on an individual level (e.g., a study by the authors of the present study; Friehs \& Frings, 2018), but it is unknown whether this process can be disrupted. In the present study we therefore investigate whether tDCS can not only be used to increase but also to decrease the efficiency of the $\operatorname{cog}$ nitive response inhibition process. 


\section{The stop-signal task and its neural underpinnings}

Although Lappin and Eriksen (1966) invented the task, the term stop-signal task (SST) was coined later by Logan, Cowan, and Davis (1984). Participants performing the SST are required to react to a stimulus (e.g., left or right pointing arrow) and on a random subset of trials a stop signal (e.g., a coloration of the arrow) is presented, which requires participants to withhold their response. The latency between the onset of the initial go signal and the stop signal is called stop-signal delay (SSD). Depending on the duration of the SSD, the SST will either get more difficult (i.e., if SSD increases) or easier (i.e., if SSD decreases). In a typical SST, the SSD is continuously adjusted on a trial-by-trial basis to provoke an error rate of 50\% (Band, van der Molen, \& Logan, 2003; Logan, Schachar, \& Tannock, 1997). From a participant's performance data, the stop-signal reaction time (SSRT) can be calculated. The SSRT is an estimate for the covert inhibition process (for a detailed account of SSRT calculation, see Verbruggen et al., 2013, 2019). Specifically, the SSRT describes the average time needed for an individual to reach the "point of no return" - the moment after which the ballistic phase of the action has progressed far enough as to where it can no longer be stopped. An SSRT of around 200 milliseconds was shown to be the typical duration of the inhibition process in young, healthy adults (Logan, 2015).

A task such as the SST requires the cancelation of a prepotent response - more specifically, an inhibition of an already initiated action. FMRI studies probing the neurological underpinnings of the SST report that there are several brain areas involved-specifically, the dorsolateral prefrontal cortex (DLPFC), inferior frontal cortex (IFC), dorsal anterior cingulate cortex (ACC), and the presupplementary motor area (preSMA; for review, see Aron, Robbins, \& Poldrack, 2004). Recent fMRI studies specified the area of activation and report a more right-lateralized PFC activity; especially during the stop process (e.g., Hughes et al., 2014; Hughes, Fulham, Johnston, \& Michie, 2012; Hughes, Johnston, Fulham, Budd, \& Michie, 2013; Xue, Aron, A\& Poldrack, 2008). ${ }^{1}$ Supporting this claim, imaging studies on tasks related to the SST, such as the go/no-go, report a mostly righthemispheric prefrontal activation (Garavan, Ross, Murphy, Roche, \& Stein, 2002; Menon, Adleman, White, Glover, \& Reiss, 2001).

Furthermore a study by Swann et al. (2012) on a rare patient with subdural grid coverage of cortical regions showed that the preSMA was active before the right IFC during stopping. This is in line with the proposed role of the PFC in the perception-action cycle (Fuster, 1988, 2017; Miller \& Cohen,

\footnotetext{
${ }^{1}$ For a detailed summary on fMRI studies dealing with the SST, see Friehs and Frings (2018)
}

2001), where the PFC is responsible for providing goaldirected feedback to motor systems and does not act independently. This feedback loop may result in the PFC being able to select the appropriate response and exert inhibitory control whenever needed. Taken together, previous research consistently suggests that the rDLPFC is essential for cognitive influences on response inhibition.

\section{Cathodal tDCS and its effects on response inhibition}

Cathodal tDCS is usually associated with an inhibition of the underlying brain area. Generally, tDCS can be applied online or offline. Online stimulation refers to co-occurring stimulation and task performance by the participant. offline stimulation describes a stimulation, where tDCS is applied for usually up to $20 \mathrm{~min}$ before the to-be-modulated task. Magnetic resonance spectroscopy (MRS) provides evidence that cathodal offline tDCS (i.e., stimulation before the to-be-modulated task) causes a reduction in glutamatergic neuronal activity and long-term depression-like changes within the cortex (Stagg \& Nitsche, 2011). Long-term depression depends on the association between presynaptic and postsynaptic activity, mostly on glutamatergic synapses and the NMDA receptor. Since cathodal tDCS reduces the glutaminergic activity, it leads to a lower release of glutamate into the synapses, which in turn weakens the postsynaptic depolarization (Cooke \& Bliss, 2006). And even though the neurophysiological evidence strongly suggests that a modulation of brain functioning using cathodal tDCS is possible, little is known about the actual effects of cathodal tDCS on cognition (e.g., Berryhill, 2014; Jacobson, Koslowsky, \& Lavidor, 2012; Schroeder \& Plewnia, 2017).

Although there have been a number of studies that set out to modulate SST performance via tDCS, very few have implemented a cathodal tDCS condition. Hsu et al. (2011) report no significant modulation of inhibitory functions after online cathodal tDCS of the preSMA. Similarly, no effect of cathodal stimulation on SST performance was reported by studies using offline tDCS over the right inferior frontal gyrus (or, most recently, Stramaccia et al., 2015). Interestingly, in a study utilizing online tDCS, neither anodal nor cathodal stimulation affected SSRT (Stramaccia, Penolazzi, Altoè, \& Galfano, 2017). Furthermore, some studies investigated the effects of cathodal stimulation on tasks similar to the SST, such as an anticipation timing task with a motor component (HaydukCosta, Drummond, \& Carlsen, 2013), a cognitive reflection task (Oldrati, Patricelli, Colombo, \& Antonietti, 2016), or a go/no-go task (Lapenta, Fregni, Oberman, \& Boggio, 2012). Hayduk-Costa, Drummond, and Carlsen (2013) report that cathodal stimulation of the SMA had no effect on inhibition, but contrary to their hypothesis, anodal tDCS led to a 
decreased inhibition process. Conversely, cathodal tDCS over the DLPFC affected performance in a cognitive reflection task, as indicated by an increase in impulsive, wrong responses (Oldrati, Patricelli, Colombo, \& Antonietti, 2016). Lastly, bilateral temporal cortex stimulation seems to affect go/no-go task performance in a polarity-specific and genderspecific way (Lapenta, Fregni, Oberman, \& Boggio, 2012). Against the background of literature, it seems reasonable to state that cathodal tDCS effects on (response) inhibition are inconsistent. The inconsistency of cathodal tDCS effects is mirrored in the contemporary state of research on tDCS effects on cognition in general. Partly, this inconsistency might stem from widely different study procedures (e.g., electrode placement, electrode size, current density, stimulation timing).

In fact, there seems to be a lack of studies implementing a cathodal tDCS procedure at all. There may be several reasons why cathodal tDCS studies are seldom conducted or published as compared with anodal ones. First, it has been suggested that inhibitory cathodal tDCS effects are less pronounced and harder to find (probability of finding a cathodal-inhibition effect: $48 \%$ ) compared with enhancing anodal tDCS effects (probability of finding an anodalexcitation effect: 81\%; Zheng, Alsop, \& Schlaug, 2011; for review, see Berryhill, 2014; Jacobson et al., 2012). The authors speculated this might be because the inhibitory cathodal effects sometimes cannot generate sufficient inhibition in the targeted brain area if the initial brain state is already high. Second, cathodal tDCS effects can be contradictory. Even though cathodal stimulation has been mostly associated with inhibitory effects on the brain, there is evidence that it could even enhance cognition under certain circumstances. There are a lot of factors being discussed as a reason for why cathodal tDCS might enhance cognition - for example, improved signal-tonoise-ratio or reduction of network activity in hyperactive regions (for review, see Schroeder \& Plewnia, 2017).

As a result, cathodal tDCS is underresearched, although investigating the effect of cathodal tDCS is crucial to our understanding of tDCS modulation of cognition. Ideally, one could modulate a cognitive process (i.e., a specific experimental effect) by anodal and cathodal tDCS stimulation in a contrary manner, thereby dissociating facilitative and inhibitory processes.

\section{The present study}

The present study set out to decrease performance in the SST via the use of cathodal tDCS. Specifically, we hypothesized that by manipulation of the prefrontal cortex the cognitive inhibition process (as measured by SSRT) should be affected. Any modulation of other response and performance measures due to tDCS (e.g., no-signal RT) would lead us to conclude that a motor process was also affected by the stimulation. To this end, an improved study procedure was used, compared with former attempts to measure tDCS effects on the SST. We aimed to maximize stimulation focality by using a comparatively small electrode placed over the rDLPFC (F4 position), while a larger electrode was positioned over the left deltoid. As a result, the current flow was most pronounced in the rDLPFC. $^{2}$ This is in line with the recommendations provided by Nitsche et al. (2008). To assess whether this electrode placement would lead to the predicted, area-specific stimulation of the rDLPFC, the current flow was simulated using HDExplore software (Version 3.0; Soterix Medical Inc, New York; see Fig. 1b). All participants had to participate in two SST sessions separated by 20 minutes of tDCS (either cathodal or sham stimulation).

Furthermore, the experimental design mimicked a study by Friehs and Frings (2018), who were able to successfully modulate SST performance via single-session offline anodal tDCS. For reasons of comparison the data of the current study (cathodal stimulation) and the published data of Friehs and Frings (2018) are pooled and analyzed together in an additional across-experiments analysis.

\section{Experiment}

\section{Method}

Sample Forty-five, right-handed students ( 33 female, 12 male) ages 19-29 years (mean age $22.02 \pm 2.53$ years) participated in the study. Handedness was determined by self-report. We only recruited right-handed participants because the reference electrode was placed extracephalically over the left deltoid and the SST required participants to react with their right hand. All participants had normal or corrected-to-normal vision. Participants were excluded from the study if information provided suggested prior neurological, psychiatric, or cardiovascular diseases. Furthermore, subjects were excluded if they recently consumed illegal drugs or large amounts of alcohol the previous night. The study was approved by the local ethics committee of the University of Trier. ${ }^{3}$ All participants provided written informed consent.

Because of our previous study on the modulation of SST performance (Friehs \& Frings, 2018), we expected an effect of $f=.33$ and a medium-sized correlation between measures of $r$ $=.5$. Together with an $\alpha$ value of .05 and a power of $1-\beta=$ .95 , a sample of at least 32 participants was planned to find a

\footnotetext{
${ }^{2}$ It cannot be ruled out other prefrontal areas adjacent to the targeted DLPFC were modulated by the stimulation as well. Chief among those are the preSMA and the right IFC, which both contribute to SST performance. We will turn to this issue in the General Discussion.

${ }^{3}$ Ethics committee, University of Trier, Universitätsring 15, D-54286, Trier, Germany.
} 


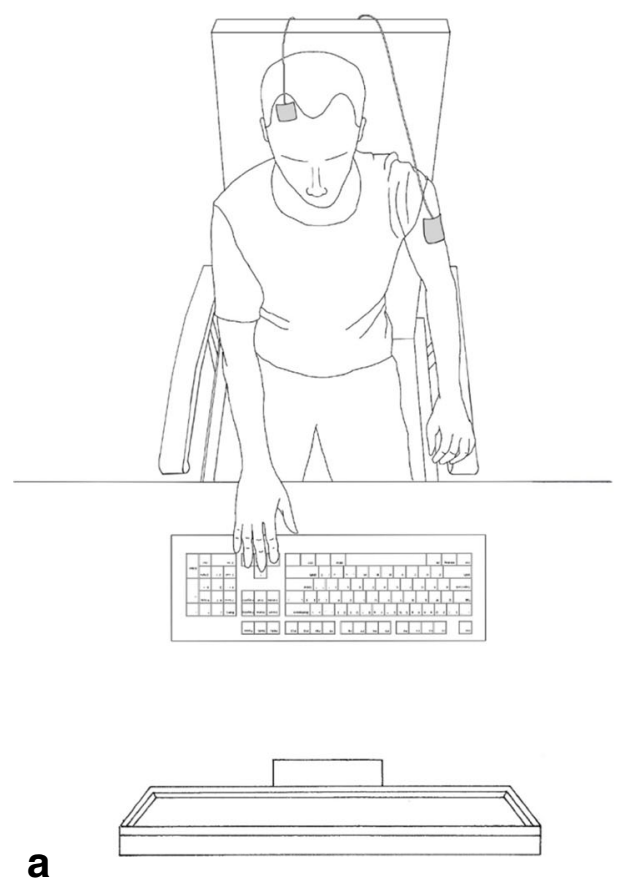

Fig. 1 Depiction of electrode position, including the calculated current flow and the trial sequence displayed during the stop-signal task. a In the cathodal as well as the sham condition, one $9 \mathrm{~cm}^{2}(3 \mathrm{~cm} \times 3 \mathrm{~cm})$ electrode was positioned over the rDLPFC (F4 position according to the extended 10-20 electrode reference system (Chatrian et al., 1988), while the $35 \mathrm{~cm}^{2}$ $(5 \mathrm{~cm} \times 7 \mathrm{~cm})$ reference electrode was applied over the left deltoid

similar effect. Calculations were carried out using $\mathrm{G}^{*}$ Power Version 3.1.3 (Faul, ErdFelder, Lang, \& Buchner, 2007).

tDCS Direct current was provided by a constant current stimulator (four-channel DC-STIMULATOR by NeuroConn, Ilmenau, Germany). In the cathodal as well as the sham condition, one $9 \mathrm{~cm}^{2}(3 \times 3 \mathrm{~cm})$ electrode was positioned over the rDLPFC (F4 position according to the extended 10-20 electrode reference system; Chatrian et al., 1988), while the 35 $\mathrm{cm}^{2}(5 \times 7 \mathrm{~cm})$ reference electrode was applied over the left deltoid muscle (see Fig. 1a). In the cathodal stimulation condition a constant current of $0.5 \mathrm{~mA}$ was applied for $19 \mathrm{~min}$. There was a ramp-up/ramp-down period of $30 \mathrm{~s}$ at the start and end of the direct current stimulation. This resulted in a current density of $0.056 \mathrm{~mA} / \mathrm{cm}^{2}$ and $0.014 \mathrm{~mA} / \mathrm{cm}^{2}$, respectively. In the sham condition, a ramp-up/ramp-down phase of 30 -s each was included at the start and right at the end of the supposed stimulation. Figure 1b depicts the calculated current flow. The stimulation was controlled via a panel PC, and current flow patterns over the stimulated brain regions could be validated using the software HD-Explore (Soterix Medical Inc., New York, NY).

Stop-signal task Participants were seated in front of a 19-inch color monitor with a viewing distance of $65 \mathrm{~cm}$ in a normally muscle. The participant was seated $65 \mathrm{~cm}$ away from the monitor. b Direct current flow during anodal stimulation using HD-Explore software (Version 3.0, Soterix Medical Inc, New York). The tDCS electrodes were positioned over F4 and the left deltoid. The MNI 152 template was used for the MRI overlay. $\mathbf{c}$ Display sequence for a trial in the employed SST. (Color figure online)

lit room. Participants responded using only their right hand by pressing one of two marked keys on a keyboard in front of the monitor. The stop-signal task (SST) was implemented using the free-of-charge STOP-IT2 MATLAB code provided by Frederick Verbruggen, which is the newer iteration of the preceding STOP-IT software (Verbruggen, Logan, \& Stevens, 2008). The pretest as well as the posttest SST consisted of three blocks of 100 trials, which contained $75 \%$ go and $25 \%$ stop trials. Between separate blocks, a pause of $15 \mathrm{~s}$ was granted. Each trial begins with the presentation of a fixation dot, which is replaced by a left-pointing or right-pointing arrow after $250 \mathrm{~ms}$. On go trials, participants had to react to a left-pointing arrow by pressing the left arrow key with their index finger, and to react to a right-pointing arrow by pressing the right arrow key with their ring finger. The stop signal, the blue coloration of the arrow, was displayed following a variable delay (the stop-signal delay; SSD), which was initially set to $250 \mathrm{~ms}$. Overall the SSD was continuously adjusted with the staircase procedure in order to obtain a probability of responding of $50 \%$. After the reaction was successfully stopped (i.e., button press was inhibited), the SSD was increased by $50 \mathrm{~ms}$, whereas when the participants did not stop successfully, the SSD was decreased by $50 \mathrm{~ms}$. The stimulus was presented for a maximum of $1,500 \mathrm{~ms}$ or until reaction. The intertrial interval was set to $500 \mathrm{~ms}$. Figure $1 \mathrm{c}$ depicts the 
display sequence for the SST. Apart from the aforementioned SSD (the probability of a correct inhibition and, most importantly, SSRT), several additional performance measures are logged and calculated. This includes two variables that are directly related to accuracy. First are the amount of omission errors (reflecting the probability of missed response on nosignal trials), and second are choice errors (reflecting the probability of a wrong response on no-signal trials). Additionally, two variables that are tied to RTs are logged: no-signal RT reflects the speed of a (correct) response on trials without a stop signal, and signal $R T$ indicates the latency of the incorrectly executed response on stop signal trials. Furthermore the probability of a correct inhibition (i.e., the likelihood of inhibiting an already initiated action) is recorded for each participant. All participants performed the SST prior to and after 20 minutes of anodal or sham tDCS.

Procedure Participants were randomly assigned to one of two tDCS conditions: (1) cathodal stimulation of the right dorsolateral prefrontal cortex or (2) sham stimulation of the right dorsolateral prefrontal cortex. Participants were naïve to the condition to which they were assigned. Each participant was subjected to a standardized procedure: fill out (1) a questionnaire concerning the exclusion criteria and demographic data; (2) pre-tDCS SST; (3) tDCS-application; (4) post-tDCS SST (identical to the pre-tDCS task); and (5) side effects questionnaire and hair cleaning. The whole experiment took around 90 minutes from entering to exiting the lab.

Design The experiment was based on a 2 (test: pre-tDCS vs. post-tDCS $) \times 2$ (tDCS stimulation: prefrontal cathodal vs. sham) mixed design with only the tDCS stimulation independent variable (IV) being varied between participants. The main dependent variable (DV) was the stop-signal reaction time (SSRT; i.e., the estimate of time needed to respond to the stop signal and to cancel the movement), which is a measure of the covert inhibition process. The estimation of the SSRT was based on the integration method described by Verbruggen et al. (2013).

Data analysis Data analysis was done in four stages. First, as described in the following data reduction section, we excluded any participant that was uncooperative or produced faulty data. Second, in a preliminary analysis stage, all remaining data were checked for satisfaction of the horse-race model. Third, we analyzed SSRT to test our hypothesis of whether or not SSRT can be modulated by cathodal tDCS. Fourth, all additionally gathered performance and side-effect measures were analyzed in order to explore any additional effects tDCS might have had.

Data reduction For the exclusion of participants, we followed the recommendations by Verbruggen and Logan (2015). First, we looked at the difference between the signal-response RT and the no-signal RT for every subject, because SSRT should not be estimated for subjects with signal-response RTs longer than no-signal RTs. The rationale behind this criterion is a direct consequence of the horse-race model, wherein a response that is executed even when a stop signal is presented has to be the result of an extremely fast process (Logan 2015; Verbruggen \& Logan, 2015). No subject had to be excluded based on this criterion in the pre-tDCS or post-tDCS session. Second, we aimed to identify outliers and participants that showed a strategic reaction during the SST (i.e., waiting for the stop signal to show and then reacting very fast). We characterized a strategic behavior as a high ratio between the overall no-signal RT and the SSRT. Based on the Tukey (1977) outlier criterion, three participants had to be excluded because of strategic behavior in the pre-tDCS and post-tDCS block. ${ }^{4}$ This resulted in a final sample of 42 subjects ( 31 female, 11 male), with a mean age of 22.07 years $(S D=2.57)$. The cathodal stimulation group was composed of 12 females and eight males (mean age $=21.80$ years, $S D=2.39$ ), while the sham stimulation group contained 19 females and three males (mean age $=22.32$ years, $S D=2.75$ ).

\section{Results}

RT means, SSDs, and SSRTs are depicted in Table 1. Errors and accuracy rates are depicted in Table 2.

To foreshadow the results, analyses focus on participants' performance in the SST, specifically the performance change from the pre-tDCS session to the post-tDCS session, depending on the stimulation group. Since our hypothesis focused on the response inhibition process, the SSRT was the focus of our analysis - specifically, we expected an increase in SSRT after cathodal tDCS, indicating a less efficient inhibition process. Additionally, error rates as well as no-signal and signal RTs were analyzed as a form of secondary analysis. Finally, control analyses were carried out. As Fig. 2 shows, offline cathodal tDCS decreased performance in the SST as evidenced by an increase in SSRT (see also Table 1). Importantly, control analyses revealed that this was not due to a general speed-up of responses after cathodal stimulation of reaction times. Furthermore, the subjective side effects of the stimulation were comparable between groups indicating successful blinding (see Table 3 in the Appendix).

Preliminary analysis To validate the gathered data Verbruggen and Logan (2015) recommend showing, that there is a statistical difference between the average signal RT and the average

\footnotetext{
${ }^{4}$ Two of the three participants stood out as outliers in the premeasurement as well as the postmeasurement. One of the three excluded participants orally admitted to strategic behavior after the pre-tDCS block and was reminded to read the instructions properly in the post-tDCS session.
} 
Table 1 Mean RTs in milliseconds (standard deviations in brackets below) dependent on time of testing and tDCS condition

\begin{tabular}{|c|c|c|c|c|c|c|c|c|}
\hline & \multicolumn{4}{|l|}{ Cathodal } & \multicolumn{4}{|l|}{ Sham } \\
\hline & Signal RT & No-signal RT & SSD & SSRT & Signal RT & No-signal RT & SSD & SSRT \\
\hline Pre & 464.45 (113.99) & $550.97(147.99)$ & $332.59(31.13)$ & $193.31(19.34)$ & $447.91(143.17)$ & $515.52(143.17)$ & $292.32(37.51)$ & $204.89(26.02)$ \\
\hline Post & 465.73 (137.64) & $548.04(137.64)$ & $322.64(36.01)$ & $211.04(21.69)$ & $461.17(181.41)$ & $512.26(208.14)$ & $295.45(45.80)$ & $201.34(32.04)$ \\
\hline
\end{tabular}

no-signal RT for each experimental condition. A MANOVA, with trial type as within-subjects factor and stimulation type as a between-subjects factor revealed that signal RT and nosignal RT are significantly different in the pre-tDCS, $F(1$, $40)=139.675, p<.001$, as well as the post-tDCS, $F(1,40)$ $=128.782, p<.001$, block, as indicated by the main effects trial type.

SSRT SSRTs were submitted to a 2 (test: pre-tDCS vs. posttDCS $) \times 2$ (tDCS: prefrontal cathodal vs. sham) MANOVA. ${ }^{5}$ Both the main effect test, $F(1,40)=2.70, p=.10$, and tDCS, $F(1,40)=0.02, p=.89$, were not significant, suggesting that SSRT was on average comparable between pre-tDCS and post-tDCS testing as well as between the two stimulation conditions (cathodal vs. sham). More importantly, the interaction between time of testing (pre-tDCS vs. post-tDCS) $\times$ Stimulation Condition (cathodal vs. sham) was significant, $F(1,40)=6.01, p<.05, \eta_{\mathrm{p}}{ }^{2}=.13$, showing that changes in SSRT vary depending on the stimulation condition (see Fig. 2 ). To confirm our hypothesis, two Bonferroni-corrected post hoc $t$ tests against zero at $p=.025$ were carried out to evaluate the SSRT change over time for each group separately. Specifically, the SSRT was significantly longer following cathodal tDCS, $t(19)=-3.14, p=.005$, but remained equal after sham stimulation, $t(21)=0.55, p=.59$. This indicates that the inhibition process significantly was impaired by cathodal stimulation of the rDLPFC. Additionally, SSRT in the pre-tDCS block was submitted to a $t$ test as to make sure that baseline performance across groups was equivalent. Results indicated that there was no statistically significant difference between the two groups, $t(40)=-1.62, p=.11$, in their baseline performance (see Table 1).

Error analysis Stop-signal task performance can cause two different types of errors: choice errors (i.e., pressing the wrong button after a go signal) and omission errors (i.e., missing a response on no-signal trials). Both types of errors were submitted separately to a 2 (test: pre-tDCS vs. post-tDCS) $\times 2$ (tDCS-condition: anodal vs. sham) repeated-measures MANOVA. First, for choice errors, the aforementioned

\footnotetext{
${ }^{5}$ For details on the use of MANOVAs for repeated-measures designs, see O'Brien and Kaiser (1985); Stevens (2009); Tabachnick and Fidell (2012); Vasey and Thayer (1987).
}

analysis did not result in a significant main effect of test, $F(1,40)=.1 .70, p=.20$, or a main effect of tDCS condition, $F(1,40)=.02, p=.89$, nor did the interaction reach significance, $F(1,40)=1.97, p=.17$. Second, for omission errors, both the main effects of test, $F(1,40)=1.60, p=.21$, and tDCS condition, $F(1,40)=.02, p=.90$, were not significant, and neither was the two-way interaction, $F(1,40)=.02, p=$ .90. See Table 2.

SSD The stop-signal delay is the delay needed between the go signal and the onset of the stop signal to produce a $50 \%$ rate. SSDs were submitted to a 2 (test: pre-tDCS vs. post-tDCS) $\times 2$ (tDCS-condition: anodal vs. sham) repeated-measures MANOVA. Neither the main effect test, nor the main effect tDCS condition, or the interaction of the two, reached statistical significance (all $F \mathrm{~s}<0$ ). This indicates that the delay needed to evoke $\sim 50 \%$ errors after a stop signal did not vary depending on time or tDCS-condition (see Table 1).

No-signal RT The no-signal RT was submitted to a 2 (test: pretDCS vs. post-tDCS) $\times 2$ (tDCS stimulation: prefrontal cathodal vs. sham) repeated-measures MANOVA. Neither the main effect test, $F(1,40)=.74, p=.79$, nor the main effect tDCS, $F(1,40)=.43, p=.52$, or the two-way interaction, $F(1$, $40)=.0002, p=.99$, reached statistical significance. This result shows that participants' overall reaction times did not change over the course of the study. See Table 1.

Signal RT The signal RT was submitted to a 2 (test: pre-tDCS vs. post-tDCS $) \times 2$ (tDCS stimulation: prefrontal cathodal vs. sham) repeated-measures MANOVA. Neither the main effect test, $F(1,40)=.48, p=.48$, nor the main effect tDCS, $F(1,40)$ $=.06, p=.81$, or the two-way interaction, $F(1,40)=.33, p=$ .57 , reached statistical significance. This result shows that participants' overall reaction times did not change over the course of the study. See Table 1.

Correct inhibition The expected proportion of successful stops in a SST is 0.5 (as represented by the variable correct inhibition. See Table 2). It has been recommended to use the arcsine square root transformation for proportion data with value ranges from 0 to 1 (Osborne, 2005, 2010). Consequently, we transformed our data before the ratio of successfully inhibited trials was submitted to a 2 (test: pre-tDCS 
Table 2 Mean error rates and accuracy in their relative proportion to the total trial count (standard deviations in brackets below) dependent on time of testing and tDCS condition

\begin{tabular}{|c|c|c|c|c|c|c|}
\hline & \multicolumn{3}{|l|}{ Cathodal } & \multicolumn{3}{|l|}{ Sham } \\
\hline & Correct inhibition & Omission error & Choice error & Correct inhibition & Omission error & Choice error \\
\hline Pre & $0.49(0.005)$ & $0.0035(0.002)$ & $0.0046(0.0014)$ & $0.49(0.005)$ & $0.0059(0.003)$ & $0.0082(0.001)$ \\
\hline Post & $0.49(0.006)$ & $0.0021(0.001)$ & $0.01(0.004)$ & $0.49(0.004)$ & $0.0042(0.002)$ & $0.008(0.003)$ \\
\hline
\end{tabular}

vs. post-tDCS $) \times 2$ (tDCS stimulation: prefrontal cathodal vs. sham) MANOVA. Neither the main effect of test, $F(1,40)=$ $.06, p=.81$, nor the main effect tDCS condition, $F(1,40)=.10$, $p=.76$, or the interaction between the two, $F(1,40)=.72, p=$ .40 , reached statistical significance. These results show that the staircase procedure was successful in adjusting the SSD in a way that led to the expected and required $50 \%$ error rate.

Side effects After the experiment, the experimenters asked the subjects to fill out a side-effects questionnaire and report the intensity of certain symptoms on a visual analogue scale (coded from 0 to 100). Overall the reported side effects were small, with tingling being the most noticeable (mean $=28.98, S D=$ 23.92). All side-effect measures were submitted separately to a one-way ANOVA, with tDCS (cathodal vs. sham) as a between-subjects factor. Results show that the cathodal and sham group did not significantly differ with regard to the side effects (see Table 3 in the Appendix for a detailed summary of side-effect ratings and between-group differences).

\section{Comparison with anodal stimulation by Friehs and Frings} (2018) The data from both studies were combined in a 3
(tDCS: anodal vs. sham vs. cathodal) $\times 2$ (test: pre vs. post) MANOVA, with SSRT as the DV. Subsequently, repeated contrasts (cathodal vs. sham and anodal vs. sham) for the pre-post difference scores are reported as part of the $3 \times 2$ analysis. The main effect of test was not significant, $F(1,94)=$ $.37, p=.54$, nor was the main effect of tDCS, $F(1,94)=.004$, $p=.99$. Crucially, the interaction between time of testing (pretDCS vs. post-tDCS) $\times$ stimulation condition (anodal vs. sham vs cathodal) was significant, $F(1,94)=6.64, p<.05, \eta_{\mathrm{p}}{ }^{2}=$ .12 , showing that changes in SSRT vary depending on the stimulation condition. Repeated-contrasts analysis against the sham condition revealed that the change in SSRT was significantly different between the sham and anodal stimulation conditions (mean difference $=-19.01, S E=7.54, p<.05$ ) and a trend toward significance between sham and cathodal stimulation (mean difference $=13.93, S E=8.45, p=.10$ ). Furthermore, we tested the change in SSRT depending on the stimulation condition against zero (i.e., no change from prestimulation to poststimulation) with a $95 \%$ confidence interval. For anodal, $t(27)=2.13, p<.05$, as well as cathodal, $t(19)=3.14, p<.05$, stimulation conditions, this analysis resulted in a significant variation from zero. In stark contrast

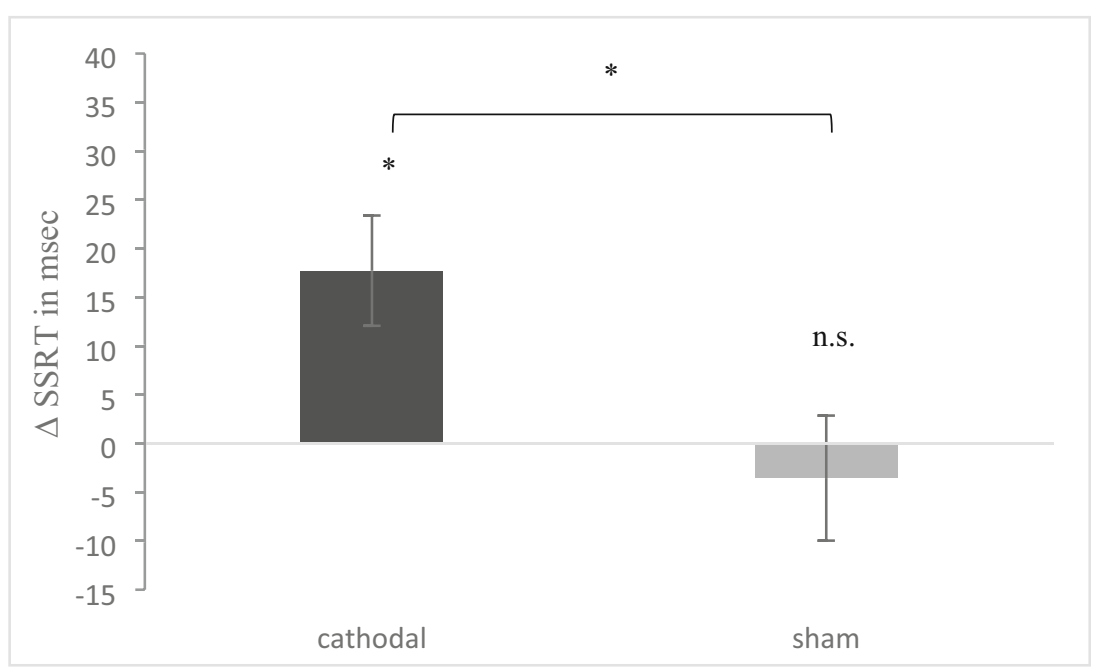

Fig. 2 Difference in SSRT (pre-tDCS vs. post-tDCS) as a function of stimulation condition (cathodal vs. sham). $* p<.05$ (two-tailed). n.s. $=$ nonsignificant difference. The bracket indicates the significant two-way interaction. The two-way interaction can also be expressed as the difference between the condition with regards to the SSRT-change over time, $t(40)=2.47, p<.05$. The result shows that cathodal tDCS led to a statistically significant increase in SSRT. Standard error of the mean are displayed for each group difference separately 
to these results, analysis on the sham stimulation condition yielded a nonsignificant result $(p=.39)$. For a visual summary of the findings, see Fig. 3.

Taken together, these results suggest that polaritydependent modulation of the response-inhibition process is possible. Anodal tDCS over the rDLPFC (F4 position) seems to decrease and cathodal stimulation increase the SSRT.

Cross-experimental control analysis Additionally, baseline SSRT was entered into a univariate ANOVA to examine potential differences between the experimental groups (anodal vs. pooled sham vs. cathodal). The baseline SSRT was comparable across all experimental groups, as was indicated by a nonsignificant main effect of condition, $F(2,95)=1.81, p=$ .17. Furthermore, both sham conditions from Study 1 and Study 2 did not differ in their baseline SSRT $(F<1, p=$ .46). Thus, it can be assumed that baseline performance across groups was comparable, and there were no large performance differences between groups.

Cross-experimental side effects Furthermore, side effects were compared across experiments (see Table 4 in the Appendix for details). Analysis revealed that there were overall no significant differences between the four experimental groups (cathodal vs. anodal - vs. sham (Study 1) vs. sham (study 2)) across the two studies.

\section{Discussion}

In this study, we aimed to explore the effects of single-session offline tDCS over the rDLPFC (F4 position) on SST performance, as to analyze whether cathodal tDCS could impair inhibition. Analysis of SSRT revealed that cathodal tDCS leads to a statistically significant increase in the second SST session (i.e., cathodal tDCS over the rDLPFC [F4 position] impaired the cognitive response inhibition process). Although cross-experimental comparisons reduced this result to a marginally significant effect $(p=.10)$. Results from control analysis support this interpretation because overall reaction times or error rates were not affected by the stimulation.

The result of the present study is partially contradictory to studies by Jacobson, Javitt, \& Lavidor, (2011) and Stramaccia et al. (2015). Both studies found no significant effect of cathodal tDCS on SST performance. Yet there are several important methodological differences between the aforementioned studies and the present one. Importantly, neither of the previous studies specifically targeted the rDLPFC, and Stramaccia et al. (2015), for example, did not implement a pre-post design. Furthermore, both studies used larger active electrodes and applied the relevant return electrode to a contralateral prefrontal area, which decreases stimulation focality. Since the prefrontal cortex is especially well interconnected, even across hemispheres (Blasi et al., 2006; Koechlin, Ody, \& Kouneiher, 2003; Miller \& Cohen, 2001), the whole prefrontal network is being stimulated, which can produce unwanted interactions between the stimulation sites (Zheng et al., 2011; Krause \& Cohen Kadosh, 2014). Consequently, we argue that the tDCS procedure in the present study presents an alternative approach that can potentially produce more unambiguous results. The lack of consistent cathodal tDCS results could be explained by a high variance in tDCS procedures and tasks. It has been argued on multiple occasions that meticulous planning can benefit tDCS studies and enhance the probability of finding a clear-cut result (Friehs \& Frings, 2018; Frings,

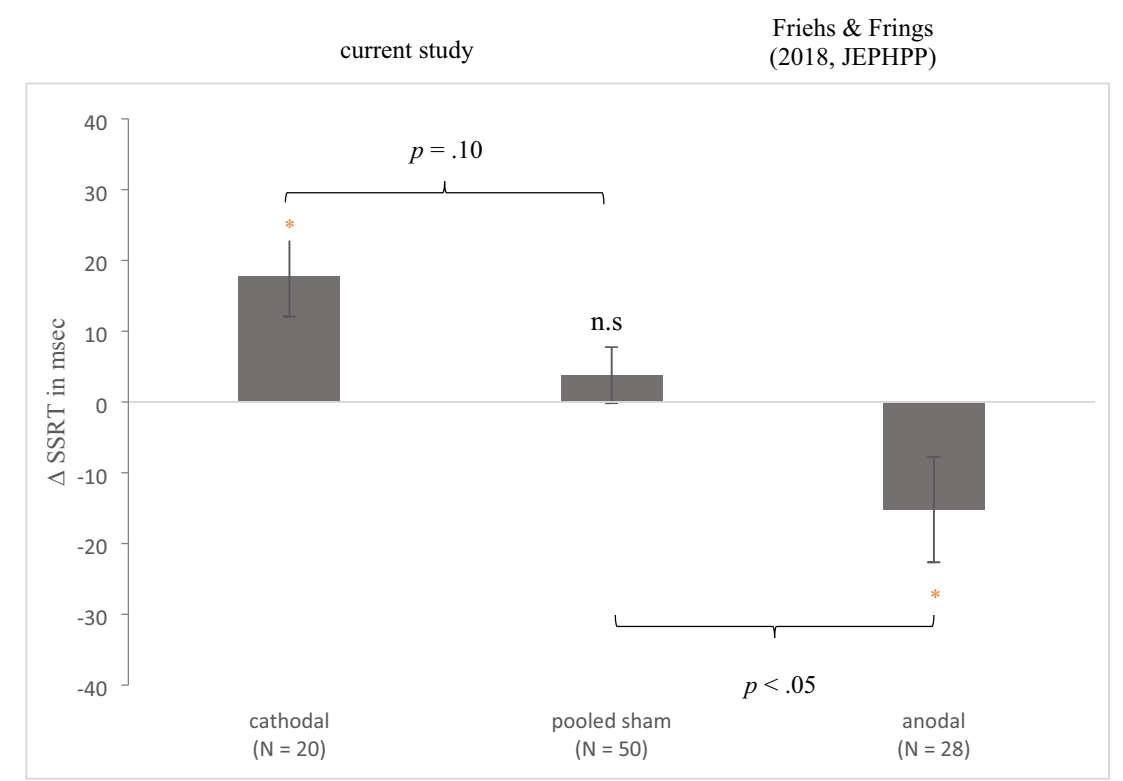

Fig. 3 Difference in SSRT (pre-tDCS vs. post-tDCS) as a function of stimulation condition (anodal vs. sham vs. cathodal). Standard error of the mean are displayed for each group difference separately. $* p<.05$ if tested against zero 
Brinkmann, Friehs, \& van Lipzig, 2018; Horvath, Carter, \& Forte, 2014; Krause \& Cohen Kadosh, 2014).

In our previous study (Friehs \& Frings, 2018), an almost identical stimulation protocol was used with the crucial difference that the anode was positioned over the rDLPFC instead of the cathode. The results of that study were twofold. First, a reduction in SSRT was observed, and second, anodal tDCS reduced the amount of omission errors. Contrarily, the present study did not find a modulation of error rates in any form, and only resulted in a significant increase of SSRT after cathodal tDCS.

\section{General discussion}

Overall results show that tDCS applied over the rDLPFC can be used to influence SST performance in a polarity-specific manner, with anodal effects being larger compared with cathodal stimulation. Specifically, an enhancement of the response inhibition process could be observed after anodal tDCS over the rDLPFC and conversely cathodal tDCS lead to an impairment of the response inhibition process; this was indicated by an increase (or decrease) in SSRT, respectively. These results provide another essential piece of evidence for the role of the DLPFC (and/or possibly adjacent regions) in inhibitory control. By up and down regulation of this brain area, only the measure for the covert inhibition process was influenced. Importantly, tDCS overall only significantly affected SSRT, which is an estimate for the covert cognitive inhibition process, and did not influence other performance measures like go RT or error rates. In the context of the horse-race model, this result provides evidence that stopping is not fixed per individual and can be influenced: Anodal tDCS can potentially postpone this moment, while cathodal tDCS could move it forward in time.

The stimulated rDLPFC is an area that is vital for cognitive control and that is particularly well connected to other brain areas, especially the preSMA (Bates \& Goldman-Rakic 1993; Goldman \& Nauta 1976; Lu, Preston, \& Strick, 1994; Schmahmann \& Pandya 1997), and which it is assumed that the preSMA receives signals from the DLPFC when a need for inhibition arises (Fuster 2015, 2017; Miller \& Cohen, 2001). The PFC therefore biases information processing in favor of task-relevant information. With regard to the SST, the stop process normally receives strengthening influences from the PFC in order to make it more competitive against the already initiated go process. With that in mind, the disordinal interaction we observed suggests that anodal stimulation improved cognitive response inhibition while cathodal stimulation disrupted the inhibition process.

Note, however, the cathodal tDCS effect was smaller in size than an anodal effect in the context of a previous study (Friehs \& Frings, 2018). Yet cathodal tDCS effects have been found to be smaller in size and have been reported as being less reliable than anodal effects (for meta-analysis, see Jacobson et al., 2012). Still, when tested against zero, the cathodal tDCS group showed a significant effect. Nevertheless, it might be important for future studies to bear in mind that anodal effects on SSRT seem to be larger than cathodal ones when replicating the cathodal tDCS effect on SST performance.

\section{Previous neuromodulation of the PFC}

The tDCS research literature is fragmented, and results widely different even if studies seem to be comparable at first glance. Take, for example, a pair of studies by Stramaccia et al. (2017; Stramaccia et al., 2015), in which tDCS was applied over the prefrontal cortex. Both studies utilized relatively similar stimulation protocols and stimulated over the $\mathrm{FC} 4$ position targeting the right IFC. Both studies implemented all stimulation conditions - anodal, cathodal, and sham-utilized 16$\mathrm{cm}^{2}$ electrodes and $1.5 \mathrm{~mA}$ (current density of $0.09 \mathrm{~mA}$ / $\mathrm{cm}^{2}$ ) but crucially stimulation timing differed. While in one an improvement after anodal offline tDCS to the right IFC was found (Stramaccia et al., 2015), the other study could not replicate those findings when utilizing online stimulation. Compared with the studies reported in this paper, there are some notable differences in tDCS procedure (current density, electrode placement, electrode size, stimulation timing), which might have contributed to the differential findings. So, concerning tDCS applications of the PFC and measuring response inhibition, it seems safe to say that further studies are warranted. Nevertheless, the general assumption that neurological states affect response inhibition processes still holds at this point in time.

Converging evidence for this claim comes from studies using another form of noninvasive brain stimulation - namely, transcranial magnetic stimulation (TMS), in conjunction with the SST. TMS originally was used to investigate the motor cortex (Barker et al., 1985), but since then, TMS has proved useful in the research of other brain areas, including the prefrontal cortex. TMS functions by applying an electromagnetic coil to the scalp either inhibits or excites a focal cortical area (Epstein, Wassermann, \& Ziemann, 2012), depending on the stimulation procedure. Similar to tDCS, not only can TMS be used to excite or inhibit a brain area, the timing of the stimulation can be varied as well. Online TMS procedures use single or paired TMS pulses to depolarize neurons and discharge an action potential (Pascual-Leone, Darvey, Rothwell, Wassermann, \& Puri, 2002), while offline TMS approaches utilize repetitive application of TMS that can produce longer-lasting aftereffects that are similar to longterm potentiation or long-term depression (Fitzgerald, Fountain, \& Daskalakis, 2006). 
With regard to the SST, TMS has been used extensively to investigate the motor component of the response inhibition process. TMS over the SMA has been shown to decrease SSRT (Obeso et al. 2017; Obeso, Cho, et al., 2013; Watanabe et al., 2015; Zandbelt, Bloemendaal, Hoogendam, Kahn, \& Vink, 2013) or increase SSRT (Cai, George, Verbruggen, Chambers, \& Aron, 2012; Chen, Muggleton, Tzeng, Hung, \& Juan, 2009; Obeso, Robles, Marrón, \& Redolar-Ripoll, 2013; Watanabe et al., 2015). With regard to the purely cognitive inhibition component, TMS has been applied over right lateralized prefrontal areas such as the IFC. Specifically, TMS over the rIFC can decrease SSRT (Obeso, Robles, Marrón, \& Redolar-Ripoll, 2013; Zandbelt et al., 2013) or increase it (Chambers et al., 2007; Verbruggen, Aron, Stevens, \& Chambers, 2010). Importantly, however, it should be mentioned that TMS compared with tDCS benefits from much higher stimulation focality, and therefore an area like the rIFC can be targeted. This is crucial because, as discussed earlier, although in the present study the stimulation was focused on the rDLPFC, we cannot rule out any secondary stimulation of the rIFC. In sum, those results provide compelling and converging evidence for the proposition that the underlying neural state (also of neuron ensembles that contribute to the cognitive part of the SSRT) is crucial for the response inhibition process.

\section{Limitations}

The present study faces several limitations. First, it relies on a cross-experimental analysis to contrast different stimulation polarities. Future studies should aim to replicate the present finding utilizing a full within-study manipulation of tDCS polarities. Second, as discussed above, the cathodal tDCS effect was not comparable in size with the anodal tDCS effect on SSRT. Third, the stimulation focality has to be addressed. Although the electrode was placed over the rDLPFC (F4 position), we cannot fully reject the notion of having stimulated-at least partly - other adjacent areas like the right IFC or pre-SMA, which have been shown to be involved in the inhibition process as well (e.g., Aron et al., 2004). As shown in Fig. 1b, the area that is most stimulated lies directly beneath the electrode positioned over the rDLPFC (F4 position), but other areas around the electrode show mild stimulation as well. This illustrates a major limitation of tDCS as compared with other noninvasive brain stimulation (NIBS) methods such as TMS. If other areas were also stimulated, even if the stimulation was weaker, then the obtained effect in this study cannot be fully attributed to a modulation of the DLPFC - in this instance, this fact has consequences for the interpretation of the underlying mechanisms (see below).

\section{The neural underpinnings of response inhibition}

It has been proposed, that the neurological state of the rDLPFC as modulated by noninvasive brain stimulation in general (e.g., tDCS or TMS) has an impact upon SST performance. In Friehs and Frings (2018), it was suggested that modulation of the rDLPFC via tDCS led to an improved monitoring process, since omission errors as well as SSRT were affected by the stimulation. This interpretation of results was in line with the suggestion that the rDLPFC is involved in monitoring the need to stop and stepping into action when inhibitory top-down control is required (Fuster, 2015). Against the background of the present results, this proposition might have to be changed, because cathodal tDCS did not influence omission errors (or any other type of error) significantly. Furthermore, the effect of anodal tDCS on omission errors was rendered insignificant in the cross-experimental comparison. Thus, it seems more reasonable to propose that tDCS can potentially affect the time at which the PFC steps into action: Anodal tDCS speeds up this process whereas cathodal tDCS delays it.

Assuming the main stimulation target was the rDLPFC, one possible explanation for the effects in both studies is tied to the role of the DLPFC in representing and implementing task rules (Aron, Robbins, \& Poldrack, 2014; Swann, Tandon, Pieters, \& Aron 2013). This is achieved actively maintaining stimulus-response associations and task goals (MacDonald, Cohen, Andrew Stenger, \& Carter, 2000; Mostofsky \& Simmonds, 2008). Moreover, it has been put forward that the DLPFC is needed for action preparation (Pochon, 2001) and whenever access to working memory is required-for example, for retrieval of a stimulus-response association (for review, see Mostofsky \& Simmonds, 2008). Building on this, the specific task rule (i.e., stopping after a stop signal) was, depending on the stimulation polarity, either strengthened or weakened in its representation and accessibility. Subsequently, since the task-goal and the stimulus-response association were either more or less present, the implementation of the stopping response was either faster or slower.

But if a substantial amount of activation spread to the rIFC - which plays a crucial role in inhibitory control ranging from suppression of memories to manual responses (Aron, Robbins \& Poldrack, 2004, 2014) - then the effect could be traced back to the modulation of the implementation of response inhibition directly. Therefore, one might also argue that tDCS applied over the rDLPFC actually increases or decreases rIFC activity and hence directly affect SSRT via suppressing of manual responses (Aron, Robbins, \& Poldrack, 2004, 2014). Note that this would also suggest a "cognitive" component of the inhibitory processes - as reaction time per se was not modulated by tDCS. This is in line with the description of the rIFC as a "brake" that can be turned on in response to an external stop signal to totally suppress a 
response (Aron, Robbins, \& Poldrack, 2014). Yet this interpretation would be somewhat narrower-SSRT modulation via rIFC can be seen as a quite specific form of inhibitory control, whereas a modulation of SSRT via the rDLPFC would place response inhibition in the wider context of cognitive control processes. In the present study, however, the specific neural origin of the SSRT modulation cannot be conclusively determined.

On a more practical and behavioral level, the results suggest that tDCS can be used to modulate intraindividual performance states. It has been shown that there is an interindividual variation in inhibition processes due to psychological disorders such as ADHD (Schachar \& Logan, 1990), schizophrenia (Hoptman et al., 2004; Kiehl, Smith, Hare, \& Liddle, 2000), or posttraumatic stress disorder (PTSD; Catarino et al., 2015; Falconer et al., 2008). But in light of the present findings, it can be argued that the cognitive capacity for inhibition varies not only interindividually but also within a person depending on his or her state. Other possible state alterations that effect inhibitory control are, for example, due to acute alcohol intake (Field et al., 2010), sleep deprivation (Anderson \& Platte, 2011; Drummond, Paulus, \& Tapert, 2006), or blood pressure levels (e.g., Frings \& Domes, 2018). Importantly, however, these state alternations refer to a physiological changes, whereas tDCS does not affect the overall physiology and only modulates the neurological state of a particular target brain region. In sum, we suggest that neurological and physiological states can affect inhibition processes in healthy individuals.

\section{Conclusion}

Taken together, our results suggest that neuromodulation of the rDLPFC due to tDCS affects response inhibition processes in a polarity dependent way - anodal stimulation boosts inhibition, whereas cathodal stimulation diminishes it, although to a lesser degree. Note that while the precise mechanism and anatomical source of the present finding is unclear, the dissociation of facilitative and inhibitory effects on response inhibition confirms previous arguments on the direct link between the neural state of the rDLPFC and SSRT.

Acknowledgements We thank Stephanie Blasl for her help in creating Fig. 1. We thank the editor and reviewers for their feedback on earlier versions of the manuscript.

Open practices statement None of the experiments were preregistered. All data is uploaded to PsychArchives and can be accessed free of charge. See https://hdl.handle.net/20.500.12034/2079 or https://doi.org/10. 23668/psycharchives. 2453

\section{Appendix}

Table 3 Side effects of the tDCS procedure for cathodal and sham conditions (rated on a $0-100$ scale) as well as a comparison of between-group differences.

\begin{tabular}{|c|c|c|c|c|c|}
\hline \multirow[t]{2}{*}{ Side effect } & \multicolumn{2}{|c|}{ Cathodal tDCS } & \multicolumn{2}{|c|}{ Sham tDCS } & \multirow[t]{2}{*}{$p$ value } \\
\hline & Mean & $S D$ & Mean & $S D$ & \\
\hline Itching & 19.75 & 22.85 & 16.68 & 23.46 & .671 \\
\hline Tingling & 33.00 & 22.27 & 25.32 & 25.29 & .304 \\
\hline Headache & 4.50 & 12.76 & 6.00 & 16.78 & .748 \\
\hline Burning & 12.00 & 18.52 & 4.18 & 12.95 & .118 \\
\hline Discomfort & 11.75 & 20.86 & 7.68 & 14.37 & .462 \\
\hline
\end{tabular}

Table 4 Side effects of the tDCS procedure for all experimental conditions across experiments (rated on a $0-100$ scale). Standard deviations in brackets under the mean

\begin{tabular}{|c|c|c|c|c|c|}
\hline \multirow[t]{2}{*}{ Side effect } & \multicolumn{2}{|l|}{ Current study } & \multicolumn{2}{|c|}{ Friehs and Frings (2018) } & \multirow[t]{2}{*}{$p$ value } \\
\hline & Cathodal tDCS & sham tDCS & sham tDCS & anodal tDCS & \\
\hline Itching & $19.75(22.85)$ & $16.68(23.46)$ & $15.71(23.67)$ & 13.57 (19.67) & .822 \\
\hline Tingling & $33.00(22.27)$ & $25.32(25.29)$ & $27.90(27.13)$ & $21.96(18.87)$ & .445 \\
\hline Headache & $4.50(12.76)$ & $6.00(16.78)$ & 8.57 (17.79) & $5.00(11.39)$ & .767 \\
\hline Burning & $12.00(18.52)$ & $4.18(12.95)$ & $15.71(21.63)$ & $9.21(14.07)$ & .129 \\
\hline Discomfort & $11.75(20.86)$ & $7.68(14.37)$ & 7.14 (11.17) & $6.79(8.95)$ & .620 \\
\hline
\end{tabular}




\section{References}

Anderson, C., \& Platten, C. R. (2011). Sleep deprivation lowers inhibition and enhances impulsivity to negative stimuli. Behavioural brain research, 217(2), 463-466.

Aron, A. R., Fletcher, P. C., Bullmore, E. T., Sahakian, B. J., \& Robbins, T. W. (2003). Stop-signal inhibition disrupted by damage to right inferior frontal gyrus in humans. Nature Neuroscience, 6(2), 115116. https://doi.org/10.1038/nn1003

Aron, A. R., Robbins, T. W., \& Poldrack, R. A. (2004). Inhibition and the right inferior frontal cortex. Trends in Cognitive Sciences. https:// doi.org/10.1016/j.tics.2004.02.010

Aron, A. R., Robbins, T. W., \& Poldrack, R. A. (2014). Inhibition and the right inferior frontal cortex: One decade on. Trends in Cognitive Sciences. https://doi.org/10.1016/j.tics.2013.12.003

Band, G. P. H., van der Molen, M. W., \& Logan, G. D. (2003). Horse-race model simulations of the stop-signal procedure. Acta Psychologica, 112(2), 105-142. https://doi.org/10.1016/S0001-6918(02)00079-3

Barker, A. T., Jalinous, R., \& Freeston, I. L. (1985). Non-invasive magnetic stimulation of human motor cortex. The Lancet, 325(8437), 1106-1107.

Bates, J. F., \& Goldman-Rakic, P. S. (1993). Prefrontal connections of medial motor areas in the rhesus monkey. Journal of Comparative Neurology, 336(2), 211-228. https://doi.org/10.1002/cne. 903360205

Berryhill, M. E. (2014). Hits and misses: Leveraging tDCS to advance cognitive research. Frontiers in Psychology, 5. https://doi.org/10. 3389/fpsyg.2014.00800

Blasi, G., Goldberg, T. E., Weickert, T., Das, S., Kohn, P., Zoltick, B., . . . Mattay, V. S. (2006). Brain regions underlying response inhibition and interference monitoring and suppression. European Journal of Neuroscience, 23(6), 1658-1664. https://doi.org/10.1111/j.14609568.2006.04680.x

Cai, W., George, J. S., Verbruggen, F., Chambers, C. D., \& Aron, A. R. (2012). The role of the right presupplementary motor area in stopping action: Two studies with event-related transcranial magnetic stimulation. Journal of Neurophysiology. https://doi.org/10.1152/ jn.00132.2012

Catarino, A., Küpper, C. S., Werner-Seidler, A., Dalgleish, T., \& Anderson, M. C. (2015). Failing to forget: Inhibitory-control deficits compromise memory suppression in posttraumatic stress disorder. Psychological Science, 26(5), 604-616.

Chambers, C. D., Bellgrove, M. A., Gould, I. C., English, T., Garavan, H., McNaught, E., ... Mattingley, J. B. (2007). Dissociable mechanisms of cognitive control in prefrontal and premotor cortex. Journal of Neurophysiology. https://doi.org/10.1152/jn.00685.2007

Chatrian, G. E., Lettich, E., \& Nelson, P. L. (1988). Improved nomenclature for the "10\%" electrode system. American Journal of EEG Technology, 28(3), 161-163.

Chen, C. Y., Muggleton, N. G., Tzeng, O. J. L., Hung, D. L., \& Juan, C. H. (2009). Control of prepotent responses by the superior medial frontal cortex. NeuroImage. https://doi.org/10.1016/j.neuroimage. 2008.09.005

Cooke, S. F., \& Bliss, T. V. P. (2006). Plasticity in the human central nervous system. Brain, 129(7), 1659-1673. https://doi.org/10. 1093/brain/aw1082

Drummond, S. P. A., Paulus, M. P., \& Tapert, S. F. (2006). Effects of two nights sleep deprivation and two nights recovery sleep on response inhibition. Journal of Sleep Research. https://doi.org/10.1111/j. 1365-2869.2006.00535.x

Epstein, C. M., Wassermann, E. M., \& Ziemann, U. (Eds.). (2012). Oxford handbook of transcranial stimulation. Oxford, UK: Oxford University Press. https://doi.org/10.1093/oxfordhb/ 9780198568926.001 .0001
Falconer, E., Bryant, R., Felmingham, K. L., Kemp, A. H., Gordon, E., Peduto, A., \& Williams, L. M. (2008). The neural networks of inhibitory control in posttraumatic stress disorder. Journal of psychiatry \& neuroscience: JPN, 33(5), 413.

Faul, F., ErdFelder, E., Lang, A.-G., \& Buchner, A. (2007). G*Power 3.1 manual. Behavioral Research Methods, 39(2), 175-191. https://doi. org/10.3758/BF03193146

Field, M., Wiers, R. W., Christiansen, P., Fillmore, M. T., \& Verster, J. C. (2010). Acute alcohol effects on inhibitory control and implicit cognition: implications for loss of control over drinking. Alcoholism: Clinical and Experimental Research, 34(8), 1346-1352.

Fitzgerald, P. B., Fountain, S., \& Daskalakis, Z. J. (2006). A comprehensive review of the effects of rTMS on motor cortical excitability and inhibition. Clinical Neurophysiology. https://doi.org/10.1016/j. clinph.2006.06.712

Friehs, M. A., \& Frings, C. (2018). Pimping inhibition: Anodal tDCS enhances stop-signal reaction time. Journal of Experimental Psychology: Human Perception and Performance. https://doi.org/ 10.1037/xhp0000579

Frings, C., Brinkmann, T., Friehs, M. A., \& van Lipzig, T. (2018). Single session tDCS over the left DLPFC disrupts interference processing. Brain and Cognition, 120, 1-7. https://doi.org/10.1016/j.bandc. 2017.11.005

Frings, C., \& Domes, G. (2018). Distractor processing is diminished under high blood pressure in healthy subjects. Manuscript submitted for publication.

Fuster, J. (2015). The prefrontal cortex. Academic Press.

Fuster, J. M. (1988). Prefrontal cortex. In Comparative Neuroscience and Neurobiology (pp. 107-109). https://doi.org/10.1007/978-1-48996776-3 43

Fuster, J. M. (2017). Prefrontal executive functions predict and preadapt. In E. Goldberg (Ed.), Executive functions in health and disease (pp. 3-19). London, UK: Elsevier. https://doi.org/10.1016/B978-0-12803676-1.00001-5

Garavan, H., Ross, T. J., Murphy, K., Roche, R. A. P., \& Stein, E. A. (2002). Dissociable executive functions in the dynamic control of behavior: Inhibition, error detection, and correction. NeuroImage, 17(4), 1820-1829. https://doi.org/10.1006/nimg.2002.1326

Goldman, P. S., \& Nauta, W. J. H. (1976). Autoradiographic demonstration of a projection from prefrontal association cortex to the superior colliculus in the rhesus monkey. Brain Research, 116(1), 145-149. https://doi.org/10.1016/0006-8993(76)90256-0

Hayduk-Costa, G., Drummond, N. M., \& Carlsen, A. N. (2013). Anodal tDCS over SMA decreases the probability of withholding an anticipated action. Behavioural Brain Research, 257, 208-214. https:// doi.org/10.1016/j.bbr.2013.09.030

Hoptman, M. J., Ardekani, B. A., Butler, P. D., Nierenberg, J., Javitt, D. C., \& Lim, K. O. (2004). DTI and impulsivity in schizophrenia: A first voxelwise correlational analysis. NeuroReport, 15(16), 24672470. https://doi.org/10.1097/00001756-200411150-00007

Horvath, J. C., Carter, O., \& Forte, J. D. (2014). Transcranial direct current stimulation: Five important issues we aren't discussing (but probably should be). Frontiers in Systems Neuroscience, 8. https:// doi.org/10.3389/fnsys.2014.00002

Hsu, T. Y., Tseng, L. Y., Yu, J. X., Kuo, W. J., Hung, D. L., Tzeng, O. J., . . . Juan, C. H. (2011). Modulating inhibitory control with direct current stimulation of the superior medial frontal cortex. NeuroImage, 56(4), 2249-2257. https://doi.org/10.1016/j.neuroimage.2011.03. 059

Hughes, M. E., Budd, T. W., Fulham, W. R., Lancaster, S., Woods, W., Rossell, S. L., \& Michie, P. T. (2014). Sustained brain activation supporting stop-signal task performance. European Journal of Neuroscience, 39(8), 1363-1369. https://doi.org/10.1111/ejn.12497

Hughes, M. E., Fulham, W. R., Johnston, P. J., \& Michie, P. T. (2012). Stop-signal response inhibition in schizophrenia: Behavioural, event-related potential and functional neuroimaging data. 
Biological Psychology, 89(1), 220-231. https://doi.org/10.1016/j. biopsycho.2011.10.013

Hughes, M. E., Johnston, P. J., Fulham, W. R., Budd, T. W., \& Michie, P. T. (2013). Stop-signal task difficulty and the right inferior frontal gyrus. Behavioural Brain Research, 256, 205-213. https://doi.org/ 10.1016/j.bbr.2013.08.026

Jacobson, L., Javitt, D. C., \& Lavidor, M. (2011). Activation of inhibition: diminishing impulsive behavior by direct current stimulation over the inferior frontal gyrus. Journal of Cognitive Neuroscience, 23(11), 3380-3387.

Jacobson, L., Koslowsky, M., \& Lavidor, M. (2012). TDCS polarity effects in motor and cognitive domains: A meta-analytical review. Experimental Brain Research. https://doi.org/10.1007/s00221-0112891-9

Kiehl, K. A., Smith, A. M., Hare, R. D., \& Liddle, P. F. (2000). An eventrelated potential investigation of response inhibition in schizophrenia and psychopathy. Biological Psychiatry, 48(3), 210-221. https:// doi.org/10.1016/S0006-3223(00)00834-9

Koechlin, E., Ody, C., \& Kouneiher, F. (2003). The architecture of cognitive control in the human prefrontal cortex. Science (New York, N.Y.), 302(5648), 1181-1185. https://doi.org/10.1126/science. 1088545

Kramer, A. F., Hahn, S., Cohen, N. J., Banich, M. T., McAuley, E., Harrison, C. R., . . . Colcombe, A. (1999). Ageing, fitness and neurocognitive function. Nature, 400(6743), 418

Krause, B., \& Cohen Kadosh, R. (2014). Not all brains are created equal: the relevance of individual differences in responsiveness to transcranial electrical stimulation. Frontiers in Systems Neuroscience, 8. https://doi.org/10.3389/fnsys.2014.00025

Lapenta, O. M., Fregni, F., Oberman, L. M., \& Boggio, P. S. (2012). Bilateral temporal cortex transcranial direct current stimulation worsens male performance in a multisensory integration task. Neuroscience Letters, 527(2), 105-109. https://doi.org/10.1016/j. neulet.2012.08.076

Lappin, J. S., \& Eriksen, C. W. (1966). Use of a delayed signal to stop a visual reaction-time response. Journal of Experimental Psychology. https://doi.org/10.1037/h0021266

Lijffijt, M., Kenemans, J. L., Verbaten, M. N., \& Van Engeland, H. (2005). A meta-analytic review of stopping performance in attention-deficit/ hyperactivity disorder: Deficient inhibitory motor control? Journal of Abnormal Psychology, 114(2), 216-222. https://doi. org/10.1037/0021-843X.114.2.216

Logan, G. D. (2015). The point of no return: A fundamental limit on the ability to control thought and action. Quarterly Journal of Experimental Psychology, 68(5), 833-857. https://doi.org/10.1080/ 17470218.2015.1008020

Logan, G. D., Cowan, W. B., \& Davis, K. A. (1984). On the ability to inhibit simple and choice reaction time responses: A model and a method. Journal of Experimental Psychology: Human Perception and Performance, 10(2), 276-291. https://doi.org/10.1037/00961523.10.2.276

Logan, G. D., Schachar, R. J., \& Tannock, R. (1997). Impulsivity and inhibitory control. Psychological Science, 8(1), 60-64. https://doi. org/10.1111/j.1467-9280.1997.tb00545.x

Lu, M.-T, Preston, J. B., \& Strick, P. L. (1994). Interconnections between the prefrontal cortex and the premotor areas in the frontal lobe. Journal of Comparative Neurology, 341(3), 375-392. https://doi. org/10.1002/cne. 903410308

MacDonald, A. W., Cohen, J. D., Andrew Stenger, V., \& Carter, C. S. (2000). Dissociating the role of the dorsolateral prefrontal and anterior cingulate cortex in cognitive control. Science. https://doi.org/10. 1126/science.288.5472.1835

Menon, V., Adleman, N. E., White, C. D., Glover, G. H., \& Reiss, A. L. (2001). Error-related brain activation during a go/nogo response inhibition task. Human Brain Mapping, 12(3), 131-143. Retrieved from http://med.stanford.edu/content/dam/sm/scsnl/documents/ Menon Error-Related Brain 01.pdf

Miller, E. K., \& Cohen, J. D. (2001). An integrative theory of prefrontal cortex function. Annual Review of Neuroscience, 24(1), 167-202. https://doi.org/10.1146/annurev.neuro.24.1.167

Mostofsky, S. H., \& Simmonds, D. J. (2008). Response inhibition and response selection: Two sides of the same coin. Journal of Cognitive Neuroscience. https://doi.org/10.1162/jocn.2008.20500

Nitsche, M. A., Cohen, L. G., Wassermann, E. M., Priori, A., Lang, N., Antal, A., . . Pascual-Leone, A. (2008). Transcranial direct current stimulation: State of the art 2008. Brain Stimulation. https://doi.org/ 10.1016/j.brs.2008.06.004

Obeso, I., Cho, S. S., Antonelli, F., Houle, S., Jahanshahi, M., Ko, J. H., \& Strafella, A. P. (2013). Stimulation of the pre-SMA influences cerebral blood flow in frontal areas involved with inhibitory control of action. Brain Stimulation. https://doi.org/10.1016/j.brs.2013.02.002

Obeso, I., Robles, N., Marrón, E. M., \& Redolar-Ripoll, D. (2013). Dissociating the role of the pre-SMA in response inhibition and switching: A combined online and off-line TMS approach. Frontiers in Human Neuroscience. https://doi.org/10.3389/fnhum. 2013.00150

Obeso, I., Wilkinson, L., Teo, J. T., Talelli, P., Rothwell, J. C., \& Jahanshahi, M. (2017). Theta burst magnetic stimulation over the pre-supplementary motor area improves motor inhibition. Brain Stimulation. https://doi.org/10.1016/j.brs.2017.05.008

O'brien, R. G., \& Kaiser, M. K. (1985). MANOVA method for analyzing repeated measures designs: an extensive primer. Psychological bulletin, 97(2), 316

Oldrati, V., Patricelli, J., Colombo, B., \& Antonietti, A. (2016). The role of dorsolateral prefrontal cortex in inhibition mechanism: A study on cognitive reflection test and similar tasks through neuromodulation. Neuropsychologia, 91, 499-508. https://doi.org/ 10.1016/j.neuropsychologia.2016.09.010

Osborne, J. (2005). Notes on the use of data transformations. Practical assessment, research and evaluation, 9(1), 42-50.

Osborne, J. W. (2010). Improving your data transformations: Applying the Box-Cox transformation. Practical Assessment, Research \& Evaluation, 15(12), 1-9.

Pascual-Leone, A., Darvey, N., Rothwell, J., Wassermann, E., \& Puri, B. (2002). Handbook of transcranial magnetic stimulation. Retrieved from https://www.ncbi.nlm.nih.gov/pmc/articles/PMC193984/

Pochon, J.-B. (2001). The role of dorsolateral prefrontal cortex in the preparation of forthcoming actions: An fMRI Study. Cerebral Cortex. https://doi.org/10.1093/cercor/11.3.260

Schachar, R., \& Logan, G. D. (1990). Impulsivity and inhibitory control in normal development and childhood psychopathology. Developmental psychology, 26(5), 710.

Schmahmann, J. D., \& Pandya, D. N. (1997). Anatomic organization of the basilar pontine projections from prefrontal cortices in rhesus monkey. Journal of Neuroscience, 17(1), 438-458.

Schroeder, P. A., \& Plewnia, C. (2017). Beneficial effects of cathodal transcranial direct current stimulation (tDCS) on cognitive performance. Journal of Cognitive Enhancement, 1(1), 5-9. https://doi. org/10.1007/s41465-016-0005-0

Stagg, C. J., \& Nitsche, M. A. (2011). Physiological basis of transcranial direct current stimulation. Neuroscientist, 17(1), 37-53. https://doi. org/10.1177/1073858410386614

Stevens, J. P. (2009). Applied multivariate statistics for the social sciences (5th ed.). New York, NY: Routledge.

Stramaccia, D. F., Penolazzi, B., Altoè, G., \& Galfano, G. (2017). TDCS over the right inferior frontal gyrus disrupts control of interference in memory: A retrieval-induced forgetting study. Neurobiology of Learning and Memory. https://doi.org/10.1016/j.nlm.2017.07.005

Stramaccia, D. F., Penolazzi, B., Sartori, G., Braga, M., Mondini, S., \& Galfano, G. (2015). Assessing the effects of tDCS over a delayed response inhibition task by targeting the right inferior frontal gyrus 
and right dorsolateral prefrontal cortex. Experimental Brain Research, 233(8), 2283-2290. https://doi.org/10.1007/s00221-0154297-6

Swann, N. C., Cai, W., Conner, C. R., Pieters, T. A., Claffey, M. P., George, J. S., . . . Tandon, N. (2012). Roles for the presupplementary motor area and the right inferior frontal gyrus in stopping action: Electrophysiological responses and functional and structural connectivity. NeuroImage, 59(3), 2860-2870. https://doi. org/10.1016/j.neuroimage.2011.09.049

Swann, N. C., Tandon, N., Pieters, T. A., \& Aron, A. R. (2013). Intracranial electroencephalography reveals different temporal profiles for dorsal- and ventro-lateral prefrontal cortex in preparing to stop action. Cerebral Cortex. https://doi.org/10.1093/cercor/bhs245

Tabachnick, B. G. (2012). Fidell, 1. S.(1996) Using multivariate statistics.

Tsai, C.-L. (2009). The effectiveness of exercise intervention on inhibitory control in children with developmental coordination disorder: Using a visuospatial attention paradigm as a model. Research in Developmental Disabilities. https://doi.org/10.1016/j.ridd.2009.05. 001

Tukey, J. W. (1977). Exploratory data analysis. analysis (Vol. 2). https:// doi.org/10.1007/978-1-4419-7976-6

Vasey, M. W., \& Thayer, J. F. (1987). The Continuing Problem of False Positives in Repeated Measures ANOVA in Psychophysiology: A Multivariate Solution. Psychophysiology. https://doi.org/10.1111/j. 1469-8986.1987.tb00324.x

Verbruggen, F., Aron, A. R., Stevens, M. A., \& Chambers, C. D. (2010). Theta burst stimulation dissociates attention and action updating in human inferior frontal cortex. Proceedings of the National Academy of Sciences. https://doi.org/10.1073/ pnas. 1001957107

Verbruggen, F., Aron, A. R., Band, G. P., Beste, C., Bissett, P. G., Brockett, A. T., \& Colzato, L. S. (2019). A consensus guide to capturing the ability to inhibit actions and impulsive behaviors in the stop-signal task. Elife, 8, e46323. https://doi.org/10.7554/eLife. 46323

Verbruggen, F., Chambers, C. D., \& Logan, G. D. (2013). Fictitious inhibitory differences: how skewness and slowing distort the estimation of stopping latencies. Psychological science, 24(3), $352-362$.

Verbruggen, F., \& Logan, G. D. (2015). Evidence for capacity sharing when stopping. Cognition, 142, 81-95. https://doi.org/10.1016/j. cognition.2015.05.014

Verbruggen, F., Logan, G. D., \& Stevens, M. A. (2008). STOP-IT: Windows executable software for the stop-signal paradigm. Behavior Research Methods, 40(2), 479-483. https://doi.org/10. 3758/BRM.40.2.479

Wang, C. H., Chang, C. C., Liang, Y. M., Shih, C. M., Chiu, W. S., Tseng, P., . . Juan, C. H. (2013). Open vs. closed skill sports and the modulation of inhibitory control. PLOS ONE, 8(2). https://doi.org/ 10.1371/journal.pone.0055773

Watanabe, T., Hanajima, R., Shirota, Y., Tsutsumi, R., Shimizu, T., Hayashi, T., Terao, Y., . . Konishi, S. (2015). Effects of rTMS of pre-supplementary motor area on fronto basal ganglia network activity during stop-signal task. Journal of Neuroscience. https://doi. org/10.1523/JNEUROSCI.3761-14.2015

Xue, G., Aron, A. R., \& Poldrack, R. A. (2008). Common neural substrates for inhibition of spoken and manual responses. Cerebral Cortex, 18(8), 1923-1932. https://doi.org/10.1093/cercor/bhm220

Zandbelt, B. B., Bloemendaal, M., Hoogendam, J. M., Kahn, R. S., \& Vink, M. (2013). Transcranial magnetic stimulation and functional MRI reveal cortical and subcortical interactions during stop-signal response inhibition. Journal of Cognitive Neuroscience. https://doi. org/10.1162/jocna00309

Zheng, X., Alsop, D. C., \& Schlaug, G. (2011). Effects of transcranial direct current stimulation (tDCS) on human regional cerebral blood flow. NeuroImage, 58(1), 26-33. https://doi.org/10.1016/j. neuroimage.2011.06.018

Publisher's note Springer Nature remains neutral with regard to jurisdictional claims in published maps and institutional affiliations. 